\title{
HIGH RESOLUTION MRI BRAIN IMAGE SEGMENTATION TECHNIQUE USING HOLDER EXPONENT
}

\author{
M. Ganesh ${ }^{1}$ and Dr. V. Palanisamy ${ }^{2}$ \\ ${ }^{1}$ ECE, Info Institute of Engineering, Anna University, Chennai, India \\ mganeshtvm1719@gmail.com \\ ${ }^{2}$ Principal, Info Institute of Engineering, Anna University, Chennai, India \\ vpsamyin@gmail.com
}

\begin{abstract}
Image segmentation is a technique to locate certain objects or boundaries within an image. Image segmentation plays a crucial role in many medical imaging applications. There are many algorithms and techniques have been developed to solve image segmentation problems. Spectral pattern is not sufficient in high resolution image for image segmentation due to variability of spectral and structural information. Thus the spatial pattern or texture techniques are used. Thus the concept of Holder Exponent for segmentation of high resolution medical image is an efficient image segmentation technique. The proposed method is implemented in Matlab and verified using various kinds of high resolution medical images. The experimental results shows that the proposed image segmentation system is efficient than the existing segmentation systems.
\end{abstract}

\section{KEYWORDS}

Holder Exponent, Gabor Filter, Clustering, Image Transformation, Morphological Operation.

\section{INTRODUCTION}

For some applications, such as image recognition or compression, we cannot process the whole image directly for the reason that it is inefficient and unpractical. Therefore, several image segmentation algorithms were proposed to segment an image before recognition or compression. Image segmentation is to classify or cluster an image into several parts (regions) according to the feature of image, for example, the pixel value or the frequency response.

Neurological conditions are the most common cause of serious disabilities and have a major, but often unrecognized, impact on health and social services. It can change the shape, volume and distribution of brain tissue. The advantages of magnetic resonance imaging (MRI) over other diagnostic image modes are its high spatial resolution and excellent discrimination of soft tissues. MRI is the preferred imaging techniques for examining neurological conditions which requires segmentation into different classes which are regarded as the best available representations for biological tissues and it can be performed by using image segmentation. However, the multispectral information of the MRI signal Kek-Shih Chuang et al. [13] has been included. Segmenting and reconstructing structures from medical image volumes into a more manageable analytic form is hindered by the sheer size of the data sets and the complexity and variability of the anatomic shapes of interest. The challenge is to extract boundary elements belonging to the DOI: $10.5121 / \mathrm{ijsc} .2012 .3404$ 
same structure and integrate these elements into a coherent and consistent model of the structure Tim McInerney et al. [12].

Spectral pattern is not sufficient in high resolution image for image segmentation due to variability of spectral and structural information. Thus the spatial pattern or texture techniques are used. Thus the concepts of Holder Exponent for segmentation in high resolution image are used. Holder Exponent is basically used to measure the local regularity of image.

Local Binary Patterns is a technique that describes the texture in terms of both statistical and structural characteristics. Holder Exponent is used to assess the roughness or smoothness around each pixel of the image. The measure of dispersion is used to compute the Holder Exponent.

The window size is assessed to detect the localized singularities. Larger window size is insensitive to noise that leads to loss of information of singularity, while the smaller window size represent the singularity well but sensitive to noise. So it is preferable to determine the window from two respects.

- Additional singularity should not be contained in the same window

- The size of the window should be enlarged on the location without obvious singularity.

An iterative clustering procedure is adapted to detect the range of cluster contained in the kernel, localize the cluster center (this approach moves the range of holder exponent values in the direction where the density is higher), and identify the cluster contained in the kernel (background, range). A clustering procedure including maximum likelihood analysis is used to classify the Holder Exponent image.

\section{A SURVEY ON RECENT RESEARCHES}

Voorons et al. [7] to the corresponding objective functions enhances their insensitiveness to noise to some extent, they still lack enough robustness to noise and outliers, especially in absence of prior knowledge of the noise; 2) In their objective functions, there exists a crucial parameter $\alpha$ used to balance between robustness to noise and effectiveness of preserving the details of the image, it was selected generally through experience; 3) The time of segmenting an image is dependent on the image size, and hence the larger the size of the image, the more the segmentation time.

Weiling Cai et al. [10] proposed a work by incorporating local spatial and gray information together, a novel fast and robust FCM framework for image segmentation Dao-Qiang Zhang et al. [11], i.e. Fast Generalized Fuzzy c-means clustering algorithms (FGFCM), was proposed in their work. FGFCM can mitigate the disadvantages of FCM_S and at the same time enhances the clustering performance. Furthermore, FGFCM not only includes many existing algorithms, such as fast FCM and Enhanced FCM as its special cases.

Mohamad Awad et al. [9] proposed a new multi-component image segmentation method using a nonparametric unsupervised artificial neural network called Kohonen's self-organizing map (SOM) and hybrid genetic algorithm (HGA). SOM was used to detect the main features that were present in the image; then, HGA was used to cluster the image into homogeneous regions without any a priori knowledge. 
Christoph Rhemann et al. [2] presented a new approach to the matting problem which splits the task into two steps: interactive trimap extraction followed by trimap-based alpha matting. By doing so they have gained considerably in terms of speed and quality and were able to deal with high resolution images. The database was used to train their system and to validate that both their trimap extraction and their matting method improve on state-of-the-art techniques.

Roger Trias-Sanz et al. [3] have reviewed several existing colour space transformations and textural features, and investigate which combination of inputs gives best results for the task of segmenting high-resolution multispectral aerial images of rural areas into its constituent cartographic objects such as fields, orchards, forests, or lakes, with a hierarchical segmentation algorithm. A method to quantitatively evaluate the quality of hierarchical image segmentation was presented, and the behaviour of the segmentation algorithm for various parameter sets was also explored.

A.E. Dorr et al. [6] described a three-dimensional atlas of the mouse brain, manually segmented into 62 structures, based on an average of $32 \mu \mathrm{m}$ isotropic resolution T2-weighted, within skull images of forty 12 week old C57B1/6J mice, scanned on a $7 \mathrm{~T}$-scanner. Individual scans were normalized, registered, and averaged into one volume. Structures within the cerebrum, cerebellum, and brainstem were painted on each slice of the average MR image while using simultaneous viewing of the coronal, sagittal and horizontal orientations.

T. Esch et al. [5] an optimization approach is proposed to minimize over- and under segmentations in order to attain more accurate segmentation results using Definiens Developer software. The procedure aims at the minimization of over- and under segmentations in order to attain more accurate segmentation results. The optimization iteratively combines a sequence of multiscale segmentation, feature-based classification, and classification-based object refinement. The developed method has been applied to various remotely sensed data and was compared to the results achieved with the established segmentation procedures provided by the Definiens Developer software.

Frederic Galland et al. [4] proposed a new and fast unsupervised technique for segmentation of high-resolution synthetic aperture radar (SAR) images into homogeneous regions. That technique was based on Fisher probability density functions of the intensity fluctuations and on an image model that consists of a patchwork of homogeneous regions with polygonal boundaries. The segmentation was obtained by minimizing the stochastic complexity of the image. Different strategies for the pdf parameter estimation were analyzed, and a fast and robust technique was proposed. Finally, the relevance of that approach was demonstrated on high-resolution SAR images.

Debasish Chakraborty et al. [8] proposed a measure to compute the Holder exponent (HE) to assess the roughness or smoothness around each pixel of the image. The localized singularity information was incorporated in computing the HE. An optimum window size was evaluated so that $\mathrm{HE}$ reacts to localized singularity. A two-step iterative procedure for clustering the transformed HE image was adapted to identify the range of HE, densely occupied in the kernel and to partition Holder exponents into a cluster that matches with the range. Holder exponent values (noise or not associated with the other cluster) were clubbed to a nearest possible cluster using the local maximum likelihood analysis.

Daniel Glasner et al. [1] proposed a method for detecting the high resolution locations of membranes from low depth-resolution images. They have approached that problem using both a 
method that learns a discriminative; over-complete dictionary and a kernel SVM. They have tested that approach on tomographic sections produced in simulations from high resolution

\section{IMAGE SEGMENTATION}

Images play various and significant roles in our daily life. In medicine, many diagnoses are based on biomedical images derived from x-rays, computerized tomography, ultra-sound, magnetic resonance and other imaging modalities.

In the current age of information technology, the issues of distributing images efficiently and making better use of them are of substantial concern. To achieve these goals by using computers. Images are first digitized so that computers are read them. Image processing algorithms are then applied to instruct computers to handle the images automatically.

Image segmentation is the method to divide an image into regions of different types. For example, in computer aided diagnosis, it is helpful to segment medical images into different tissues so that a specified measurement can be done automatically.

The main goal of image segmentation is to accurately extract the shape of targets from various types of medical images. Image segmentation can be approached from three different philosophical perspectives. The region approach, one assigns each pixel to a particular object or region. In the boundary approach, one attempts only to locate the boundaries that exist between the regions. In the edge approach, one seeks to identify edge pixels and then link them together to form the required boundaries.

The result of image segmentation is a set of segments that collectively cover the entire image, or a set of contours extracted from the image. Each of the pixels information is varied from colour, intensity or texture. Adjacent regions are significantly different with respect to the same characteristic(s).

\section{PROPOSED WORK}

An efficient image segmentation technique to segment the high resolution medical images. Initially, the filtering technique is applied to the query image to remove the noise content in the medical image. Then morphological operations like dilation and erosion are done over the filtered image. Finally, the image is segmented using Holder Exponent. The basic flow diagram as shown in the Fig. 1 below. 


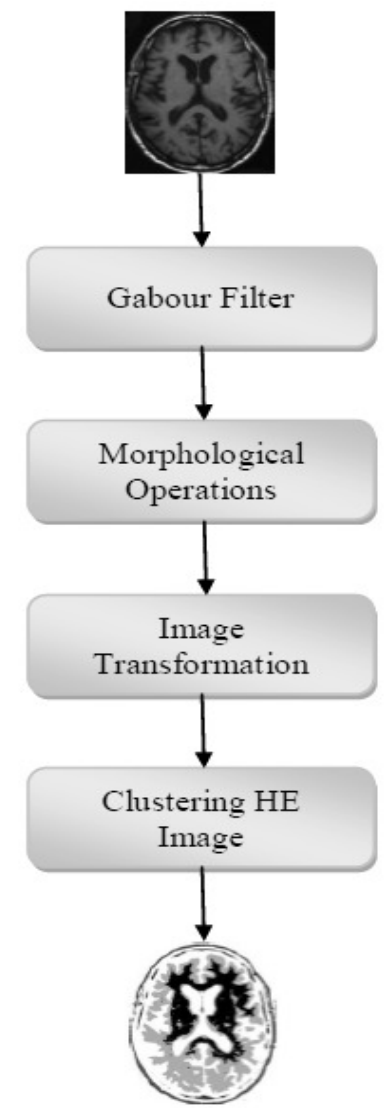

Fig. 1 Basic Flow of the Proposed System

\subsection{Gabor Filtering}

Gabor Filter, a kind of frequency filter, which has been applied to texture analysis, moving object tracking and face recognition, are also shown to be good fits in character recognition field. The primary step for high resolution image segmentation is, removing the noise from the query image using Gabor filter. This filter removes the noise content from the image and makes the image ready for the recognition. In this filter the real and imaginary component of the image has been determined and it can be represented in orthogonal directions.

The complex term of the image $g(x, y)$ can be represented as

$$
g(x, y ; \lambda, \theta, \psi, \sigma, \gamma)=\exp \left(-\frac{x^{\prime 2}+\gamma 2 y^{\prime 2}}{2 \sigma^{2}}\right) \exp \left(i\left(2 \pi \frac{x^{\prime}}{\lambda}+\psi\right)\right)
$$

The real component of the image $g(x, y)$ can be represented as

$$
g(x, y ; \lambda, \theta, \psi, \sigma, \gamma)=\exp \left(-\frac{x^{\prime 2}+\gamma 2 y^{\prime 2}}{2 \sigma^{2}}\right) \cos \left(2 \pi \frac{x^{\prime}}{\lambda}+\psi\right)
$$


The imaginary component of the image $g(x, y)$ can be represented as

$$
g(x, y ; \lambda, \theta, \psi, \sigma, \gamma)=\exp \left(-\frac{x^{\prime 2}+\gamma 2 y^{\prime 2}}{2 \sigma^{2}}\right) \sin \left(2 \pi \frac{x^{\prime}}{\lambda}+\psi\right)
$$

Where,

$$
\begin{aligned}
& x^{\prime}=x \cos \theta+y \sin \theta \\
& y^{\prime}=-x \sin \theta+y \cos \theta
\end{aligned}
$$

In this above equation, $\lambda$ represents the wavelength of the sinusoidal factor, $\theta$ represents the orientation of the normal to the parallel stripes of a Gabor function, $\psi$ is the phase offset, $\sigma$ is the sigma of the Gaussian envelope and $\gamma$ is the spatial aspect ratio.

\subsection{Morphological Operations}

Morphology is a broad set of image processing operations that process images based on shapes. Morphological operations apply a structuring element to an input image, creating an output image of the same size. In a morphological operation, the value of each pixel in the output image is based on a comparison of the corresponding pixel in the input image with its neighbors. By choosing the size and shape of the neighborhood, you can construct a morphological operation that is sensitive to specific shapes in the input image. The most basic morphological operations are dilation and erosion. Dilation adds pixels to the boundaries of objects in an image, while erosion removes pixels on object boundaries. The number of pixels added or removed from the objects in an image depends on the size and shape of the structuring element used to process the image.

The state of a given pixel in the output image is determined by the corresponding pixel and its neighbors in the input image. For Dilation, the value of the output pixel is the maximum value of all the pixels in the input pixel's neighborhood. In a binary image, if any of the pixels is set to the value 1 , the output pixel is set to 1 , and for erosion, the value of the output pixel is the minimum value of all the pixels in the input pixel's neighborhood. In a binary image, if any of the pixels is set to 0 , the output pixel is set to 0 .

\subsection{Image Transformation using HE}

The Holder exponent analysis is used here to transform the image for the identification of the texture. It does not require any prior information about the pixel intensity. The most important parameters to compute the Holder exponent is predefined measure and it is used to estimate the degree of texture around each pixel. Using this measure the smoothness or roughness around the each pixel can be assessed. Here linear regression analysis can be used to determine the measure of dispersion of pixel values.

Let the subset $\Omega *$ of the region $\Omega$ contains only those pixels which intersect the perimeter of the circle of radius $r$. Hence for $t$ number of increasing radius (i.e., $r=1$ to $t$ ) there will be $t$ number of subsets $\Omega *$. Subsequently the radius $r$ versus the intensity values $I(i)$ of that subset 
$\Omega *$ is plotted and from the least square fit of regression line calculate the intensity value $J$ for each radius $r$. As a result, a new measure $K(i)=|I(i)-J|$, for each $i \in \Omega *$ is obtained. In turn this provides the dispersion of pixels from the line of regression. The above measure can be represented as:

$$
\mu \operatorname{disp}(\Omega *)=\{K(i)=|I(i)-J| ; \operatorname{Min}(I(i)) \leq J \leq \operatorname{Max}(I(i))\}
$$

where $J$ is the derived intensity value for radius $r$ using the regression equation. The dispersion of pixels in the subset $\Omega *$ is calculated from $\mu \operatorname{disp}(\Omega *)$.

As described in the definition, for each pixel, a series of measure of balls centered on this pixel with incremental radius values are obtained. Logarithmic plots of computed measure $K$ versus radius $R$ values are drawn and got the Holder exponent $\alpha$ as follows:

$$
A=\frac{1}{n} \sum_{r=1}^{t} \sum_{i=1}^{m} \log \frac{K(i)}{R(r)}
$$

where $t$ is the total number of identified balls, $m$ is the number of intersected pixel on the perimeter of the circle of radius $R(r)$ and $N$ is the total number of pixels under each ball of radius $R(r)$.

Assessment of window size is one of the important issues to study the localized singularities. In this study, the window size is assessed to detect the localized singularities. The larger window size is insensitive to noise that leads to loss of information of singularity, while the smaller window size represents the singularity well, but sensitive to noise. So it is preferable to determine the window from two respects:

- Additional singularity should not be contained in the same window; so the window should be diminished to keep the singularity,

- The size of the window should be enlarged on the locations without obvious singularity.

Considering all the above factors, measure of dispersion of pixels from the line of regression is used here as a standard for choosing the size of window. Consequently it calculates the average dispersion of pixel values from the line of regression of ten (user defined) windows (selected randomly in the image) and determines whether the opted larger size window is small enough, i.e., the difference with the smallest average dispersion is small enough than the appointed threshold. The opted size of window is adopted for computation of localized singularities. Otherwise, it determines the smaller size of window and finds again whether the difference between the smallest average dispersion is small enough than the appointed threshold, then this size of window is adopted.

\subsection{Clustering}

After transforming the medical image using Holder Exponent, the image has to be clustered to form the segmented image. The clustering of the HE images comprises of three important steps as follows. 
- Detect the range (RQ) of a cluster contained in the kernel based on a preliminary estimated HE range,

- Localize the cluster center, and

- Identify the cluster contained in the kernel G.

The range RQ of a cluster in the Holder exponent image is defined as follows, Let us consider $G=\{g k l$, Holder exponent value in $G(k, l)\}$, where $k=1, \ldots, m$ and $l=1, \ldots, m$ is a kernel with $m^{2}$ Holder exponent (HE). $Q$ is a cluster in $G$ with center $C Q($ mean $)$. Then the range $R Q$ of the cluster $Q$ contains only those $\mathrm{HE}$ values satisfying the following properties:

$$
A b s(g k l-C Q)<R Q
$$

Eqn. 8 means that cluster $Q$ contains that range of HE value, which has a minimum degree of association (represented by $R Q$ ). A preliminary estimated $\mathrm{HE}$ range $R Q$ is computed before the assessment of optimal range of the cluster. It is a three-step procedure as follows.

- Computation of mean $(G)$,

- Computation of maximum $(G)$, and

- Estimation of preliminary HE range $=\operatorname{abs}($ computation of mean $(G)$-computation of $\operatorname{maximum}(G))$.

Localization of cluster is to find a center in the dataset where the 'density' (or number) of range of pixel values in $G$ within a range, i.e., $R Q$ is locally maximal. Primarily the cluster center is initialized with the mean $\mathrm{HE}$ values. Then we select the $\mathrm{HE}$ values within the $R Q$ from the center of $G$ (i.e., mean of $G$ ). Iteratively, the mean of this range of $\mathrm{HE}$ values is again calculated and subsequently the kernel center is moved to this mean. This approach moves the range of HE values in the direction where the density is higher.

This is implemented iteratively by decreasing $R Q$ with a constant value until absolute difference between the initial center $(C Q)$ and present center (ME) reaches the desired value (minimum difference). In the first iterations (when $R Q$ is still large) this technique moves the range of $\mathrm{HE}$ values to regions of the data where the 'global' density is higher (these regions often contain the large number of pixels). After some iteration (when $R Q$ is equal to constant value) the kernel center moves towards an actual range of HE values where the density is 'locally' higher. Convergence is reached if the kernel center remains stationary. If this does not happen within a certain number of iterations then last computed $\mathrm{ME}$ is considered as $C Q$.

The Cluster identification consists of two parts, Background and Range. Backgrounds are the HE values in the HE image not included between $(C Q-R Q)$ and $(C Q+R Q)$ values. Such $\mathrm{HE}$ values, either belongs to another cluster or do not belong to any cluster (noise; are not significantly associated with other $\mathrm{HE}$ values). HE values belonging to other clusters are not considered at the time of threshold calculation for the current cluster. Ranges are the HE values represented as $(C Q-R Q) \leq H E \leq(C Q+R Q)$. HE values belonging to the cluster are significantly correlated. 
The image so obtained after adopting previously mentioned clustering technique consists of noise. The classification accuracy of the image decreases due to inclusion of those noises. Here, a method is proposed to club those noises to a cluster that is spatially nearer and likelihood of occurrence is more. The method is a two-step procedure. In the first step, it computes the weight of each cluster residing in the kernel, while the maxi-mum weighted cluster is identified in the second step. Cluster weight is computed with the formula

$$
W\left(\text { Cluster }_{k}\right)=\frac{\text { freq }}{n * m}
$$

Where, $k$ is the number of cluster resides in the kernel. freq is the total number of HE falling in the range of $k^{\text {th }}$ cluster residing in the kernel. $W\left(\right.$ Cluster $\left._{k}\right)$ is the possibility (or weighting factor) to assign the HE value in the $k^{\text {th }}$ cluster $n$ representing the number of rows of the kernel and $m$ representing the number of columns of the kernel. Maximum weighted cluster is identified with the equation

$$
\left.\operatorname{MaxW}_{\left(\text {Cluster }_{k}\right)}\right)=\left(\sup \left\{W\left(\text { cluster }_{k}\right)\right\}, k=1, \ldots, L\right)
$$

Where, $L$ is the number of cluster contained in the kernel.

\section{RESULTS AND DISCUSSION}

In this section, the results obtained during the process of proposed medical image segmentation are discussed. Initially, Gabor filter has to be applied to the input query image to reduce the noise content in the image. Since, the segmentation has to be done in a clear image to get accurate segmented output. Fig. 2 shows the query image and also the image output of Gabor filter.

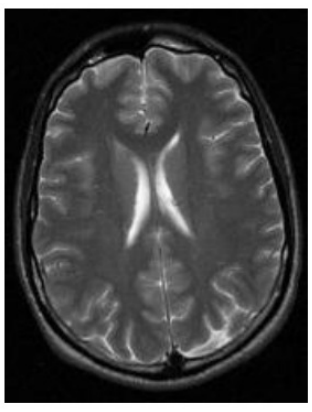

(a)

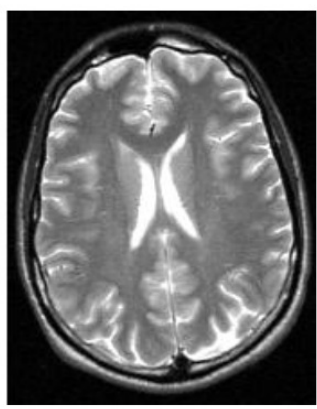

(b)

Fig. 2 (a) Input image and (b) Gabor filter output

After applying Gabor filter, the output image is subjected to morphological operations like dilation and erosion. Fig. 3 shows the output image after morphological operations. 


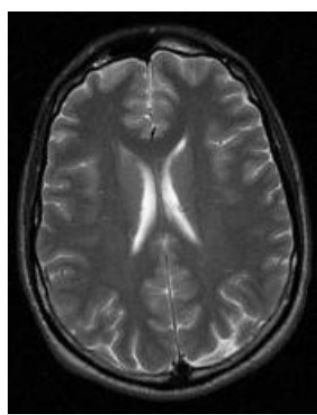

(a)

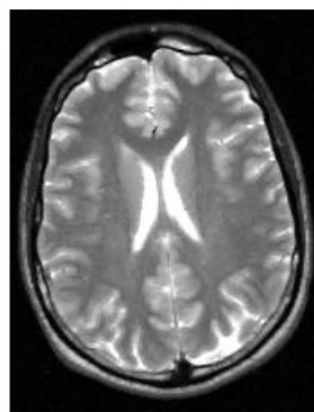

(b)

Fig. 3 (a) Input image and (b) Image after Morphological operations

Then the image is transformed using Holder exponent. In each pixel of the image the roughness or smoothness can be identified using Holder exponent. The measure of dispersion is used to compute the Holder Exponent. After image transformation, clustering is applied to cluster the image contents to form the segmented image. The segmented image has some noise content. This noise can be removed by applying the mean value for each pixel from the neighbor pixels. Thus we get the segmented output of the given medical image. Fig. 4 shows the segmented output of the medical image.

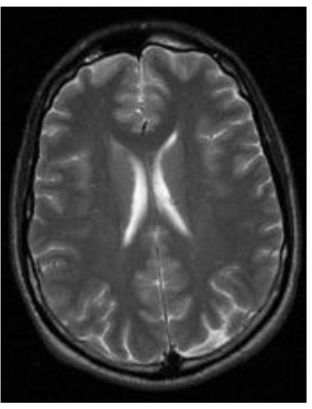

(a)

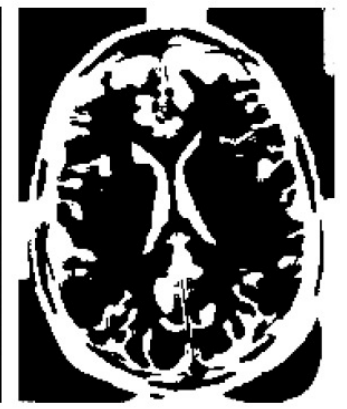

(b)

Fig. 4 (a) Input image and (b) Segmented image

\section{Comparative Analysis}

Any part of the brain show certain regions that appear whitish and others that have a darker grayish colour. These constitute the White and Grey matter respectively. Microscopic examination shows that the cell bodies of neurons are located only in grey matter which also contains dendrites and axons starting from or ending on the cell bodies. Most of the fibers within the grey matter are unmyelinated. On the other hand the white matter consists predominantly of myelinated fibers.

Grey matter is a darker colored tissue of the Central nervous system, it must be available in neuron cell bodies, and it may have the branches in dendrites and supporting cells, glia.

White matter is a paler colored tissue of the Central nervous system; it must be available in insulating material, myelin, which surrounds nerve fibers. 
International Journal on Soft Computing (IJSC) Vol.3, No.4, November 2012

Table: I Overlap measures (GM, WM) obtained for different segmentation methods

\begin{tabular}{|c|c|c|}
\hline Segmentation Method & White Matter (\%) & Grey Matter (\%) \\
\hline $\begin{array}{c}\text { Fuzzy C-Means } \\
\text { algorithm }\end{array}$ & 85.60 & 83.21 \\
\hline $\begin{array}{c}\text { Robust Fuzzy C-Means } \\
\text { Algorithm }\end{array}$ & 86.09 & 84.08 \\
\hline Proposed Method & 89.32 & 87.60 \\
\hline
\end{tabular}

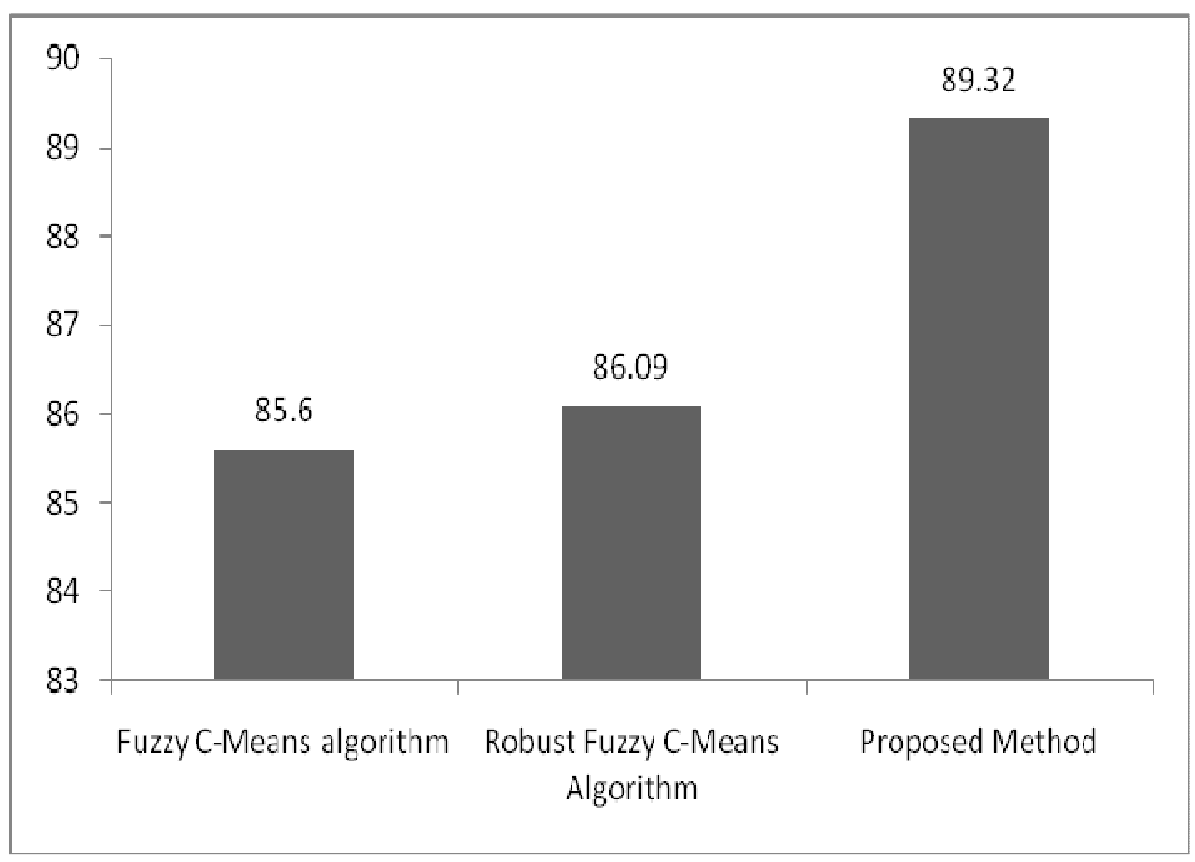

Fig. 5 White Matter comparission 


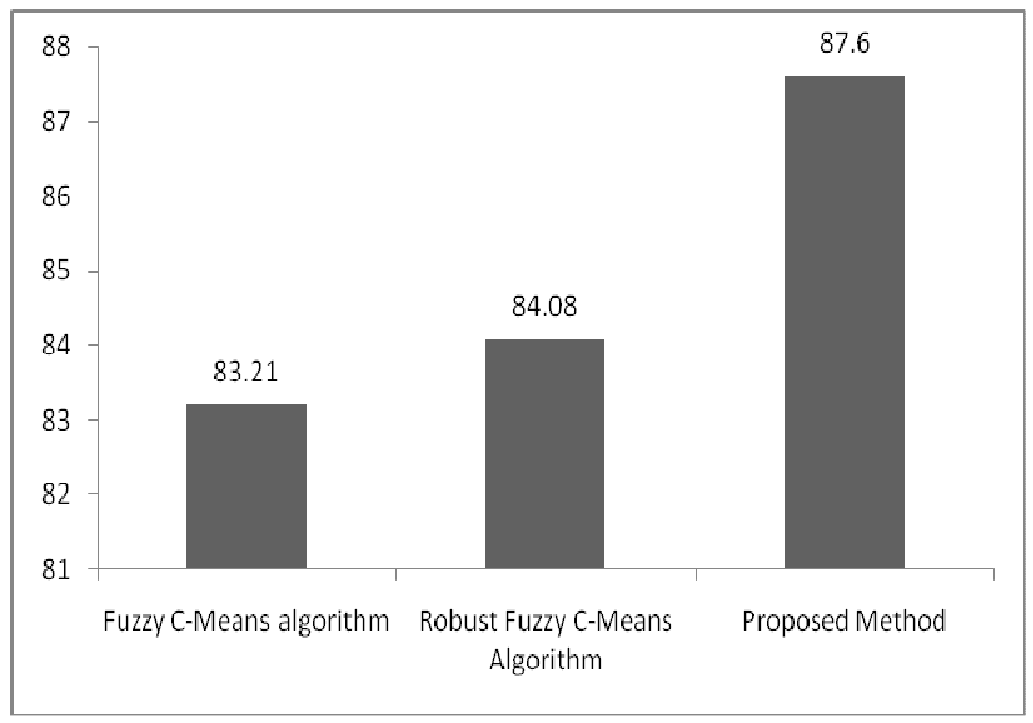

Fig. 6 Gray Matter comparission

Fig. 5 gives the graphical representation about the percentage of white matter in the MRI image and Fig. 6 gives the graphical representation about the percentage of grey matter in the MRI image. Fig. 5 and Fig. 6 shows that the proposed medical image segmentation technique is more efficient than the existing Fuzzy based segmentation since the percentage of overlap measures (WM and GM) is high as compared with the existing technique.

\section{CONCLUSION}

In recent years, For Image processing the more important technique or process is Image segmentation. In spite of the availability of a large variety of state-of art methods for brain MRI segmentation, but still, brain MRI segmentation is a challenging task and there is a need and huge scope for future research to improve the accuracy, precision and speed of segmentation methods. Here we proposed a medical image segmentation algorithm based on Holder Exponent. Since, Image Segmentation using Holder Exponent can be used to measure the roughness or smoothness around each pixel in the image, and also it does not require any prior information about the pixel intensity. Our work gives more overlap measures as compared to the existing technique, thus our medical image segmentation technique is more efficient. The proposed segmentation results shows that, the use of Holder Exponent based strategy globally leads to better results than the other state of the art methods existing now.

\section{REFERENCE}

[1] Daniel Glasner, Tao Hu, Juan Nunez-Iglesias, Lou Sche er, Shan Xu, Harald Hess, Richard Fetter, Dmitri Chklovskii, and Ronen Basri, "High Resolution Segmentation of Neuronal Tissues from Low Depth-Resolution EM Imagery," In Proc. of the 8th international conference on Energy minimization methods in computer vision and pattern recognition, pp. 261-272, 2011.

[2] Christoph Rhemann, Carsten Rother, Alex Rav-Acha, and Toby Sharp, "High Resolution Matting via Interactive Trimap Segmentation," In Proc. of the CVPR'08, 2008. 
[3] Roger Trias-Sanz, Georges Stamon, and Jean Louchet, "Using colour, texture, and hierarchial segmentation for high-resolution remote sensing," ISPRS Journal of Photogrammetry \& Remote Sensing, Vol. 63, pp. 156-168, 2008.

[4] Frederic Galland, Jean-Marie Nicolas, Helene Sportouche, Muriel Roche, Florence Tupin, and Philippe Refregier, "Unsupervised Synthetic Aperture Radar Image Segmentation Using Fisher Distributions," IEEE Tractions on Geoscience and Remote Sensing, Vol. 47, No. 8, pp. 2966-2972, Aug 2009.

[5] T. Esch, M. Thiel, M. Bock, A. Roth, and S. Dech, "Improvement of Image Segmentation Accuracy Based on Multiscale Optimization Procedure," IEEE Geoscience and Remote Sensing Letters, Vol. 5, No. 3, pp. 463-467, Jul 2008.

[6] A.E. Dorr, J.P. Lerch, S. Spring. Kabani, and R.M. Henkelmanb, "High resolution three-dimensional brain atlas using an average, magnetic resonance image of 40 adult C57B1/6J mice," Journal of Neuro Image, Vol. 42, pp. 60-69, 2008.

[7] M. Voorons, Y. Voirin, G. B. Benie, and K. Fung, "Very High Spatial Resolution Image Segmentation Based on the Multifractal Analysis," In Proc. of the $20^{\text {th }}$ ISPRS, 2004.

[8] Debasish Chakraborty, Gautam Kumar Sen, and Sugata Hazra, "High-resolution satellite image segmentation using Holder exponents," Journal of Earth System Science, Vol. 118, No. 5, pp. 609617, Oct 2009.

[9] Mohamad Awad, Kacem Chehdi, and Ahmad Nasri, "Multicomponent Image Segmentation Using a Genetic Algorithm and Artificial Neural Network," IEEE Geoscience and Sensing Letters, Vol. 4, No. 4, pp. 571-575, Oct 2007.

[10] Weiling Cai, Songcan Chen, and Daoqiang Zhang, "Fast and Robust Fuzzy C-Means Clustering Algorithms Incorporating Local Information for Image Segmentation," Journal of Pattern Recognition, Vol. 40, No. 3, pp. 825-838, Mar 2007.

[11] Dao-Qiang Zhang and Song-Can Chen, "A novel kernelized fuzzy C-means algorithm with application in medical image segmentation," Journal of Artificial Intelligence in Medicine, Vol. 32, pp. 37-50, 2004.

[12] Tim McInerney and Demetri Terzopoulos, "Medical Image Segmentation Using Topologically Adaptable Surfaces," In Proc. of the CVRMed'97, Grenoble, France, pp. 23-32, Mar 1997.

[13] Keh-Shih Chuang, Hong-Long Tzeng, Sharon Chen, Jay Wu, and Tzong-Jer Chen, "Fuzzy c-means clustering with spatial information for image segmentation," Computerized Medical Imaging and Graphics, Vol. 30, pp. 9-15, 2006.

\section{Authors}

Mani Ganesh obtained his Bachelor's degree in Electronics and Communication Engineering from Arunai Engineering College, Thiruvannamalai. Then he obtained his Master's degree in Applied Electronics from Sathyabamma University, Chennai and doing Ph.D degree in Digital Image Processing from Anna University, Coimbatore. Currently, he is a Assistant professor at the department of Electronics and Communication Enginering, INFO Institute of Engineering, Coimbatore. His specializations include Image segmentation and Enhancement for satellite Image.

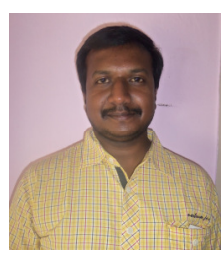

Dr. Veeraappa Gounder Palanisamy was born nearby Village at Namakkal in July 1949 and had his schooling at Namakkal. He completed his B.E. - Electronics \& Communication Engineering in the year 1972 at P.S.G. College of Technology. He completed his M.Sc. (Engg) in the field of Communication Systems at College of Engineering, Guindy, (presently Anna University, Chennai) in the year 1974. He was sponsored by Government of Tamilnadu to do his Ph.D in Communication-Antenna theory. At Indian Institute of Technology, Kharapur,West Bengal in the year 1981 and successfully completed the same. He retired as Principal, Government College of

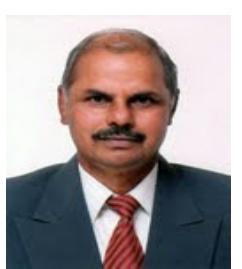
Technology, Coimbatore and presently working as Principal in Info Institute of Engineering, Coimbatore. $\mathrm{He}$ is a member in number of Academic boards, AICTE \& University inspection committee. 